\title{
Wireless and mobile sensing technologies for the future Internet
}

\author{
Abdelouahid Derhab $^{1} \cdot$ Mohamed Guerroumi $^{2} \cdot$ Mohamed Younis $^{3}$
}

Published online: 15 February 2017

(C) Institut Mines-Télécom and Springer-Verlag France 2017

Advances in wireless technologies have transformed our societies and have become an integral part of how people live and conduct business. Moreover, many of the portable computation and communication devices and personal electronic gadgets have become loaded with a variety of sensing circuitry to improve user interaction and enable context aware operation. These wireless and mobile sensing devices integrate human with their environment more than we ever thought possible. Internetworking of wireless and mobile sensing devices constitutes the concept of the future internet or the internet of things, where a large heterogeneous set of these devices is closely coupled to enable an intelligent management of many aspects of our daily activities. Example applications include environmental monitoring, healthcare, smartphone, smart grids, and smart cities The networking of these devices not only provides a flexible and robust information gathering platform but also greatly improves the reliability and efficiency of contemporary manufacturing, scientific, and military systems.

This special issue of Annals of Telecommunications is intended to provide recent advances on the use of wireless and mobile sensing technologies in the future Internet. We received 32 submissions from an open call. After a thorough reviewing process where every paper has been evaluated by at least two experts, nine papers have been conditionally accepted, subject to some revisions. Reviewers' comments were

Abdelouahid Derhab

abderhab@ksu.edu.sa

1 King Saud University, Riyadh, Saudi Arabia

2 USTHB University, Bab Ezzouar, Algeria

3 University of Maryland Baltimore County, Baltimore, MD 91361, USA very helpful first to select the most significant contribution, then to improve the content, quality, and presentation of the accepted papers. Hereafter, a summary of each paper accepted in this special issue is described.

- Fadi Al-Turjman, Mehmet Karakoc, and Melih Gunay in their paper entitled "Path Planning for Mobile DCs in Future Cities" propose a hybrid genetic-based approach for path planning for data couriers in the context of smart cities. In this work, public vehicles are utilized as data couriers to collect data from distributed access points and transmit the collect data to a central processing base station in the city. This paper covers a very useful topic related to WSN in the context of smart cities. The experimental results look promising.

- Fan Wu, Lili Xu, Saru Kumari, Xiong Li, Muhammad Khurram Khan, and Ashok Kumar Das in their paper entitled "An Enhanced Mutual Authentication and Key Agreement Scheme for Mobile User Roaming Service in Global Mobility Networks" propose an enhanced mutual authentication and a key agreement scheme for mobile user roaming service in global mobility networks. The proposed scheme has been proven to be secure and is verified using a formal security proof. Also, the proposed scheme has shown to be computationally efficient compared to existing schemes.

- Thomas Oliver Mansfield, Bogdan Ghita, and Adrian Ambroze in their paper entitled "Signals of Opportunity Geolocation Methods for Urban and Indoor Environments" propose a set of methods for improving the device geolocation estimates in indoor and urban environments without the need for additional sensing or communication hardware. Basically, the sinusoidal Kalman filter is used to enhance the performance of a RF-based geolocation system. The paper also proposes 
using the IMU sensors to further increase the localization accuracy. The proposed methods are simulated and compared to existing methods. Also, the simulation results are further validated with a set of practical tests using data link devices typical to many proposed Internet of Things (IoT) and autonomous mobile systems. The validation results show that the efficient use of signals of opportunity coupled with common navigation sensors can provide a reliable geolocation system in indoor and urban environments.

- Faouzi Sebbak and Farid Benhammadi in their paper entitled "Majority-Consensus Fusion Approach for Elderly IoT-based Healthcare Applications" develop a method for multi-sensor based activity recognition of activity of daily living (ADL) of an elderly people for an intelligent assisted-living system, which is based on the reconcilement of the evidence theory with the frequentist approach of probability calculus. This work proposes a new framework for combining evidences by merging the majority and the conjunctive rules. The authors validate their approach using simulation and show good results compared to the evidence theory and its derivatives.

- Ashfaq Ahmad, Sheeraz Ahmed, Muhammad Imran, Umar Qasim, Zahoor Ali Khan, and Nadeem Javaid in their paper entitled "On energy efficiency in underwater wireless sensor networks with cooperative routing" deal with the problem of data routing in underwater wireless sensor networks (UWSNs). The authors exploit the cooperative communication for designing an energy-efficient routing algorithm and propose a hybrid data routing protocol. The proposed protocol follows Gaussian distribution and assumes that the channel is stable for specific time interval. Channel for the acoustic link is described by path loss model in terms of frequency and distance. The authors evaluate the performances of their protocol using simulation and compare the obtained results with some existing solutions, named DBR, EEDBR, and Co-DBR.

- Zouina Doukha, Kayhan Zrar Ghafoor, Soane Ali Ben Mussa, Samira Moussaoui, and Iman Loumachi in their paper entitled "Load Balancing Aware SDMA-based Beaconing Approach in Vehicular Ad Hoc Networks" propose a SDMA-Based beaconing with load balancing for vehicular ad hoc networks. The main objective of the proposed solution is to distribute beacon transmission among all nodes within the "beacon session." The paper extends prior work of the authors in which a distributed method is developed to synchronize beacon transmissions so that all nodes proceed to their sending within a beacon session. In this work, all beacons are sent sequentially along all the period, thereby reducing the congestion.

- Shailendra Shukla, Rajiv Misra, and Abhishek Agarwal in their paper entitled "Virtual Coordinate System using Dominating Set for GPS-free Adhoc Networks" address the problem of virtual coordinate assignment in ad hoc and sensor networks. They consider sparsely connected network and propose virtual coordinate assignment technique using a graph-theoretic dominating set algorithm. The system is performed in three phases. In the first phase, it uses a Distributed Virtual Coordinate Assignment Algorithm (DVC) to identify the center node and radius of networks. In the second phase, it assigns virtual coordinates to all one-hop neighbors of the central node, and in last phase, the virtual coordinate assignment is done to the rest of nodes in the network. The system achieves good results in terms of success rate, time complexity, and message complexity.

- Fadi Al-Turjman, Melih Gunay, and Irem Kucukoglu in their paper entitled "The Road to Dynamic Future Internet via Content Characterization" investigate the issue of dynamic resource allocation in the future Internet. This work aims to efficiently manage the highly increasing data requests in information centric networks. The authors propose a multi-tier architecture, which introduces new entities between data publishers and consumers called virtual data repeaters (VDRs). These VDRs are exploited by resource reallocation process in order to alleviate the overloaded data publishers, especially in case of irregular data request patterns. Moreover, the authors propose a framework, which is based on content demand ellipse (CDE) to characterize data requests. The parameters of the CDE model the current network status and suggest efficient resource utilization based on customized data request statistics.

- Mohammed Boudali, Mustapha Reda Senouci, Mohamed Aissani, and Walid-Khaled Hidouci in their paper entitled "Activities scheduling algorithms based on probabilistic coverage models for wireless sensor networks" investigate the activity scheduling issue of wireless sensor networks used for barrier coverage, aiming to maximize the network lifetime. Three algorithms are proposed: the first one is centralized and the others are distributed. All three algorithms take into account the probabilistic sensing of nodes. In the third algorithm, the authors support fault tolerance in addition to the energy consumption. The proposed algorithms are simulated, evaluated, and compared to some existing solutions. The simulation results show that the proposed algorithms can effectively guarantee the barrier coverage and prolong the sensor network lifetime.

Acknowledgements The guest editors would like to express their deep appreciation to the editor-in-chief, Prof. Guy Pujolle, for giving the opportunity to publish this special issue, as well as the journal editorial staffs for their continuous support and guidelines during the process of this publication. The guest editors also thank all the authors for submitting quality articles and the reviewers. 